\title{
Center vortex influence on the Dirac spectrum
}

\author{
Urs Heller* \\ American Physical Society, One Research Road, Box 9000, Ridge, NY 11961-9000, USA \\ E-mail: hellereaps.org
}

\section{R. Höllwieser}

Atomic Institute, Technical University of Vienna, Wiedner Hauptstr. 8-10, A-1040 Vienna, Austria E-mail: hroman@kph.tuwien.ac.at

\section{Faber}

Atomic Institute, Technical University of Vienna, Wiedner Hauptstr. 8-10, A-1040 Vienna, Austria E-mail: faber@kph.tuwien.ac.at

\section{J. Greensite}

Physics and Astronomy Dept., San Francisco State University, San Francisco, CA 94132, USA

E-mail: jgreensite@gmail.com

\section{Š. Olejník}

Institute of Physics, Slovak Academy of Sciences, SK-845 11 Bratislava, Slovakia

E-mail: Stefan.olejnikegmail.com

\begin{abstract}
We study the influence of center vortices on the low-lying eigenmodes of the Dirac operator, in both the overlap and asqtad formulations. For center-projected configurations, one finds that the low-lying near-zero modes are present in the staggered (asqtad) formulation, but not in the overlap and "chirally-improved" formulations. We argue that this is due to the absence of a fieldindependent chiral symmetry in the latter formulations, when the Dirac operator is evaluated on the very rough configurations generated by center projection. We also confirm and extend the results of Kovalenko et al. [Phys. Lett. B 648, 383 (2007)], finding strong correlations between center vortex locations, and the scalar density of low-lying Dirac eigenmodes on unprojected lattices, in both asqtad and overlap formulations. It is found that the low-lying eigenmodes have their largest concentrations in point-like regions, rather than on submanifolds of higher dimensionality.
\end{abstract}

The XXVI International Symposium on Lattice Field Theory

July 14 - 19, 2008

Williamsburg, Virginia, USA

* Speaker. 


\section{Introduction}

Center vortices were introduced to explain quark confinement, and there are good reasons to believe that a force strong enough to confine quarks must also break chiral symmetry spontaneously [1]. Several years ago, however, Gattnar et al. [2] reported a puzzling result concerning the lowlying eigenvalue spectrum of a chirally-improved version of the Dirac operator [3], when evaluated on center projected lattices. Despite the confining properties of such lattices, these authors found a large gap in the spectrum around zero eigenvalue. This implies, via the Banks-Casher relation [凹], a vanishing chiral condensate, and unbroken chiral symmetry. In the present work we suggest that the large gap found in the chirally-improved and overlap Dirac operators, when evaluated on confining, center-projected configurations, is related to the way in which chiral symmetry is realized on the lattice. The Casher argument [1] for chiral symmetry breaking $(\chi S B)$ is based on the usual $S U\left(N_{f}\right)_{L} \times S U\left(N_{f}\right)_{R}$ symmetry of the continuum theory with massless fermions, with symmetry transformations that are independent of the gauge-field configuration. For overlap fermions, however, the chiral symmetry transformations are gauge-field independent only for configurations which vary smoothly at the lattice scale, while for the chirally-improved Dirac operator due to Gattringer, chiral symmetry itself is absent for non-smooth configurations. Center-projected configurations are as far from smooth as possible. Thus, for the overlap and chirally-improved Dirac operators evaluated on these configurations, the direct connection to continuum chiral symmetry is lost, and the Casher argument for spontaneous chiral symmetry breaking need not apply.

In section 2 we display the spectra of the overlap [5] and asqtad [6] Dirac operators, when evaluated on normal, vortex-only (i.e. center-projected), and vortex-removed lattices. The asqtad operator has a field-independent remnant chiral symmetry, and the eigenvalue gap disappears. Our results support the view that center vortices alone can induce both confinement and chiral symmetry breaking. ${ }^{1}$ In section 3 we report on other correlations between center vortex location, and the density distribution of low-lying Dirac eigenmodes, following the earlier work by Kovalenko et al. [10]. These correlations are consistent with the picture advocated by Engelhardt and Reinhardt [1], in which topological charge is concentrated at points where vortices either intersect, or twist about themselves ("writhe") in a certain way. Dirac zero modes are concentrated where the topological charge density is large, and therefore one would expect that the densities of low-lying eigenmodes would be peaked in point-like regions. We provide supporting evidence for this type of concentration. We work throughout with lattices generated by lattice Monte Carlo simulation of the tadpole improved Lüscher-Weisz pure-gauge action [12], mainly at coupling $\beta_{L W}=3.3$ (lattice spacing $a=0.15 \mathrm{fm}$ ) for the $S U(2)$ gauge group. Center projection is carried out after fixing to the direct maximal center gauge.

\section{Thin Vortices and Near-Zero Modes}

Fig. 1 displays the first twenty overlap eigenvalue pairs for a $16^{4}$ lattice at $\beta_{L W}=3.3$. There is a large gap around zero for the center-projected data, which implies zero chiral condensate. In this case we see only five distinct eigenvalue pairs. This is due to the fact that in center projection with $U_{\mu}(x)= \pm \mathbb{1}_{2}$, the two colors decouple and the eigenvalue equation $D \psi_{n}=\lambda_{n} \psi_{n}$ is invariant

\footnotetext{
${ }^{1}$ For related results, cf. Alexandrou et al. [7], Gubarev et al. [8], and Bornyakov et al. [9].
} 

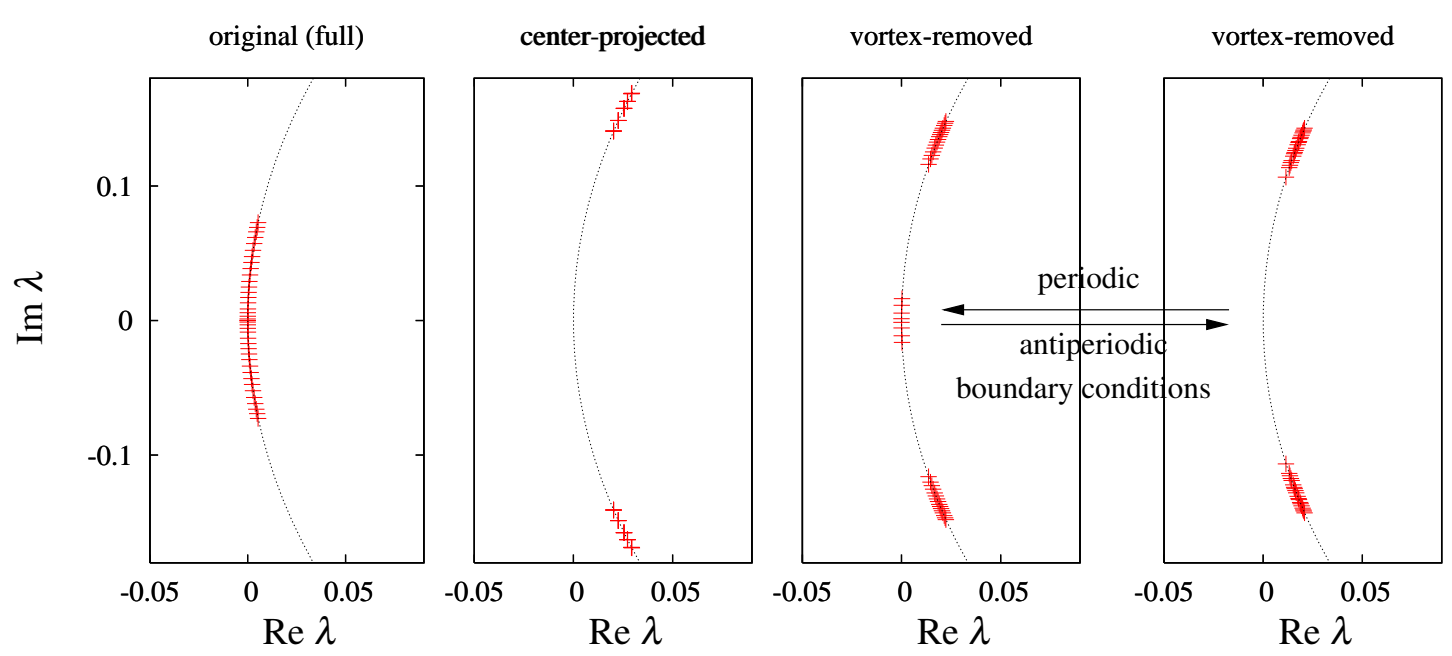

Figure 1: The first twenty overlap Dirac eigenvalue pairs on the Ginsparg-Wilson circle for a $16^{4}$ lattice at $\beta_{L W}=3.3$. The center-projected configurations show a four-fold degeneracy. Zero-modes in vortex-removed configurations disappear for antiperiodic boundary conditions.

under charge conjugation. ${ }^{2}$ A four-fold degeneracy results. The vortex-removed data shows four near-zero modes for each chirality. These correspond in number to the zero modes of the free theory, independent of lattice size, and disappear when antiperiodic boundary conditions are imposed. They are irrelevant to $\chi S B$. We have argued that the reason for the large gap in the vortex-only case is the lack of smoothness of center-projected lattices, which results in a strong field-dependence of the chiral symmetry of the Dirac operator, in contrast to the symmetry of the continuum theory. If this is indeed the reason for the gap, then the gap should disappear, and $\chi S B$ should be recovered, under a suitable smoothing of the center-projected lattice. We therefore perform an interpolation between full and projected configurations, reducing separation in the group manifold between each link variable $U_{\mu}(x)$ in maximal center gauge, and its nearest center element, by some fixed percentage. In Fig. 目 we show the low-lying eigenvalues for partial projections together with the unprojected and center-projected lattices. We see that there is no really obvious gap in the partially-projected lattices, even at $85 \%$ projection. This agrees with our conjecture that applying the overlap operator to a smoother version of the vortex-only vacuum would give a result consistent with $\chi S B$ and the Banks-Casher relation. Staggered and asqtad fermions, on the other hand, do not require a smooth configuration to preserve a subgroup of the usual continuum $S U\left(N_{f}\right)_{L} \times S U\left(N_{f}\right)_{R}$ symmetry, and by the Casher argument [1] one would expect this remaining symmetry to be spontaneously broken by any confining gauge configuration. Indeed, Ref. [7] already reported that $\langle\bar{\psi} \psi\rangle>0$ for staggered fermions on a center-projected lattice.

Fig. 3 shows the first twenty asqtad eigenvalue pairs. The low-lying eigenmode density actually increases for center-projected compared to unmodified lattices; the gap found in the overlap and chirally-improved formulations has disappeared. Thus, for the asqtad operator, we have found exactly what was expected prior to the results of Gattnar et al. [2]]: the vortex excitations of the vortex-only lattice carry not only the information about confinement, but are also responsible for $\chi S B$ via the Banks-Casher relation. The vortex-removed data develops a central band around

\footnotetext{
${ }^{2}$ This assumes that the Dirac operator has the Wilson or overlap (but not staggered) form. Thus, if $\psi_{n}$ is an eigenstate with eigenvalue $\lambda_{n}$, then $C^{-1} \psi_{n}^{*}$ is also an eigenstate, with the same eigenvalue 13.
} 


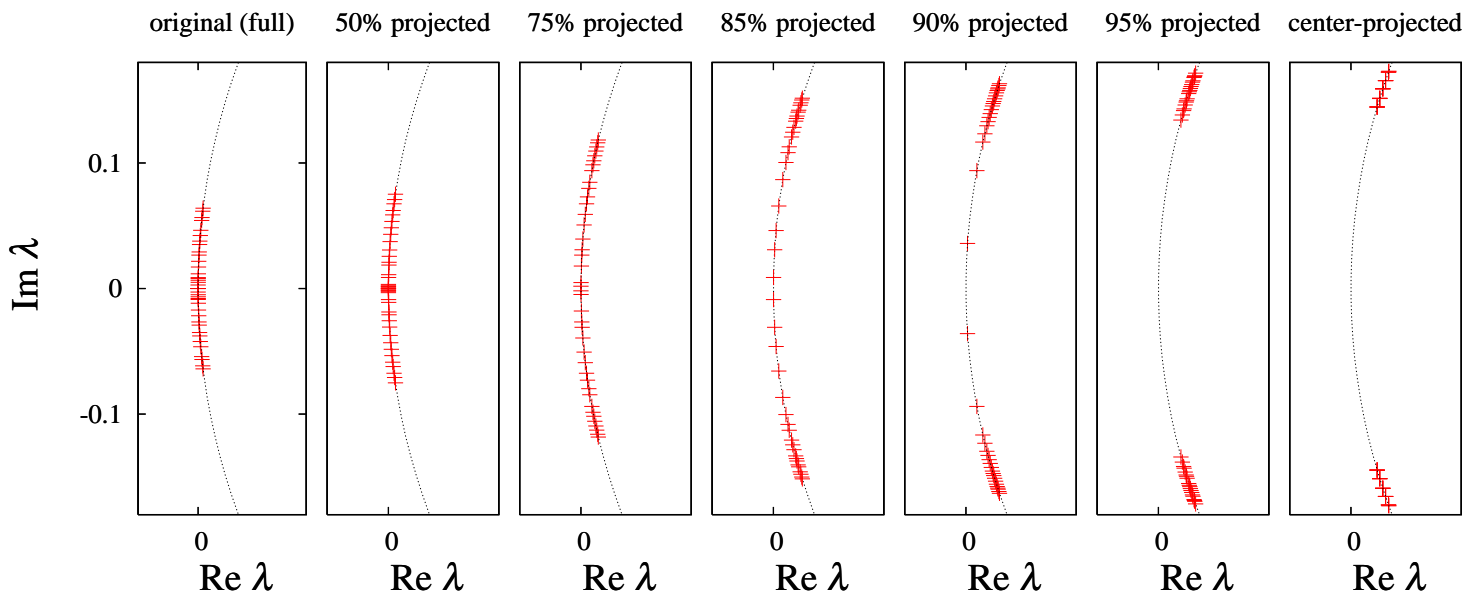

Figure 2: The first twenty overlap Dirac eigenvalue pairs from a single configuration on a $16^{4}$ lattice, antiperiodic boundary conditions at $\beta_{L W}=3.3$, for interpolated fields.

$\operatorname{Im} \lambda=0$ of eight doubly degenerate eigenmodes per chirality, which are a remnant of the 32 freefield zero modes (four zero modes for each of four "tastes" times two colors), and play no role in $\chi S B$. These modes again disappear using antiperiodic boundary conditions in one direction.
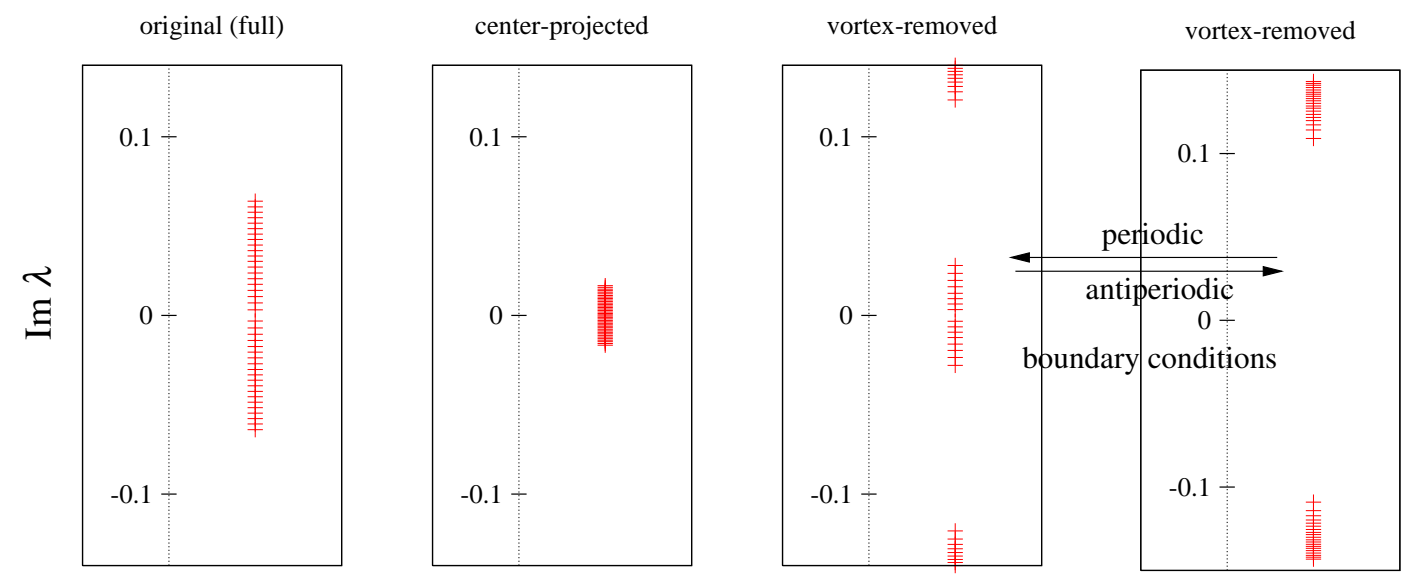

Figure 3: The first twenty asqtad Dirac eigenvalue pairs from a $16^{4}$ lattice at $\beta_{L W}=3.3$. The centerprojected configurations show no gap around zero. Zero-modes in vortex-removed configurations disappear for antiperiodic boundary conditions.

\section{Vortex surfaces and Dirac eigenmode densities}

In order to clarify the role of the vortices in the topological structure of the vacuum, we study the correlation between the density $\rho_{\lambda}(x)$ of an eigenmode with eigenvalue $\lambda$, and the number of vortex plaquettes (identified via center projection) which meet at a site. This is relevant because of the picture advanced by Engelhardt and Reinhardt [11], in which topological charge is associated with vortex intersections $\left(N_{\mathrm{v}}=8\right)$ and "writhings" $\left(N_{\mathrm{v}}=6\right)$. Following Kovalenko et al. [10], we 
define the correlator

$$
C_{\lambda}\left(N_{\mathrm{v}}\right)=\frac{\sum_{p_{i}} \sum_{x \in H}\left(V \rho_{\lambda}(x)-1\right)}{\sum_{p_{i}} \sum_{x \in H} 1} .
$$

Here the sum is over sites $p_{i}$ on the dual lattice which belong to $N_{v}$ plaquettes on the vortex surface (as identified from center projection); $V$ is the lattice volume. At each such vortex site on the dual lattice there is a second sum $(x \in H)$ over sites in a hypercube on the original lattice surrounding $p_{i}$. In Fig. $\$$ we display the data for $C_{\lambda}\left(N_{\mathrm{v}}\right)$ vs. $N_{\mathrm{v}}$ computed for eigenmodes of the asqtad Dirac operator in the full and center-projected configurations. We find that the values of $C_{\lambda}\left(N_{\mathrm{v}}\right)$ obtained from eigenmodes in the full configurations are only about a factor of four smaller than the corresponding values in the center-projected configurations, and the figures look much the same. The most important feature, in our opinion, is the fact that the correlator increases steadily with increasing number of the vortex plaquettes $N_{\mathrm{v}}$. The eigenmode density seems to be significantly enhanced at vortex sites with large $N_{\mathrm{v}}$. Our results for eigenmodes of the overlap operator are similar, and consistent with the results reported by Kovalenko et al. in Ref. [10].
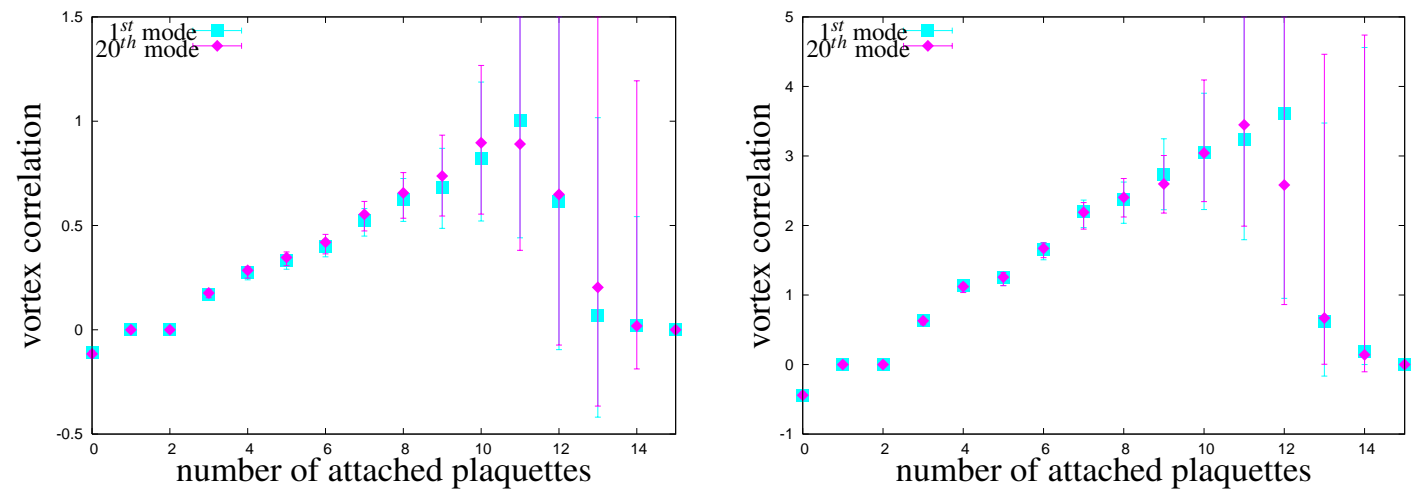

Figure 4: Vortex correlation $C_{\lambda}\left(N_{\mathrm{v}}\right)$ for asqtad staggered eigenmodes on a $20^{4}$ lattice at $\beta_{L W}=3.3$, full (left) and center-projected (right) configurations.

The correlations shown provide some degree of evidence that low-lying Dirac eigenmodes concentrate preferentially at regions on the center vortex surface where there are self-intersections or "writhing"-points, in agreement with the general picture advanced by Engelhardt and Reinhardt [11]. It is then natural to ask whether there is any supporting evidence that the eigenmode density is especially concentrated in point-like regions. To check this, we simply inspect sample plots of $\rho_{\lambda}(x)$ throughout the lattice volume. In Fig. 5 we display our data for the lowest eigenmode of the asqtad Dirac operator, in some two-dimensional slices of the four-dimensional lattice volume taken in the neighborhood of the point where $\rho_{\lambda}(x)$ is largest. Each lattice, unprojected (left) and centerprojected (right), contains several sharp peaks of this kind; the concentration of eigenmode density is in a point-like region, rather than being spread over a submanifold of higher dimensionality. Figure 6 shows the same type of data for a zero mode of the overlap Dirac operator on $16^{4}$ lattices. For full configurations the eigenmode density again is concentrated in a point-like region. For the overlap we have already noted that the spectrum evaluated in center-projected configurations is unrelated to $\chi S B$, and indeed, instead of having a sharp peak, the eigenmode concentration in this case extends over most of the lattice volume. 

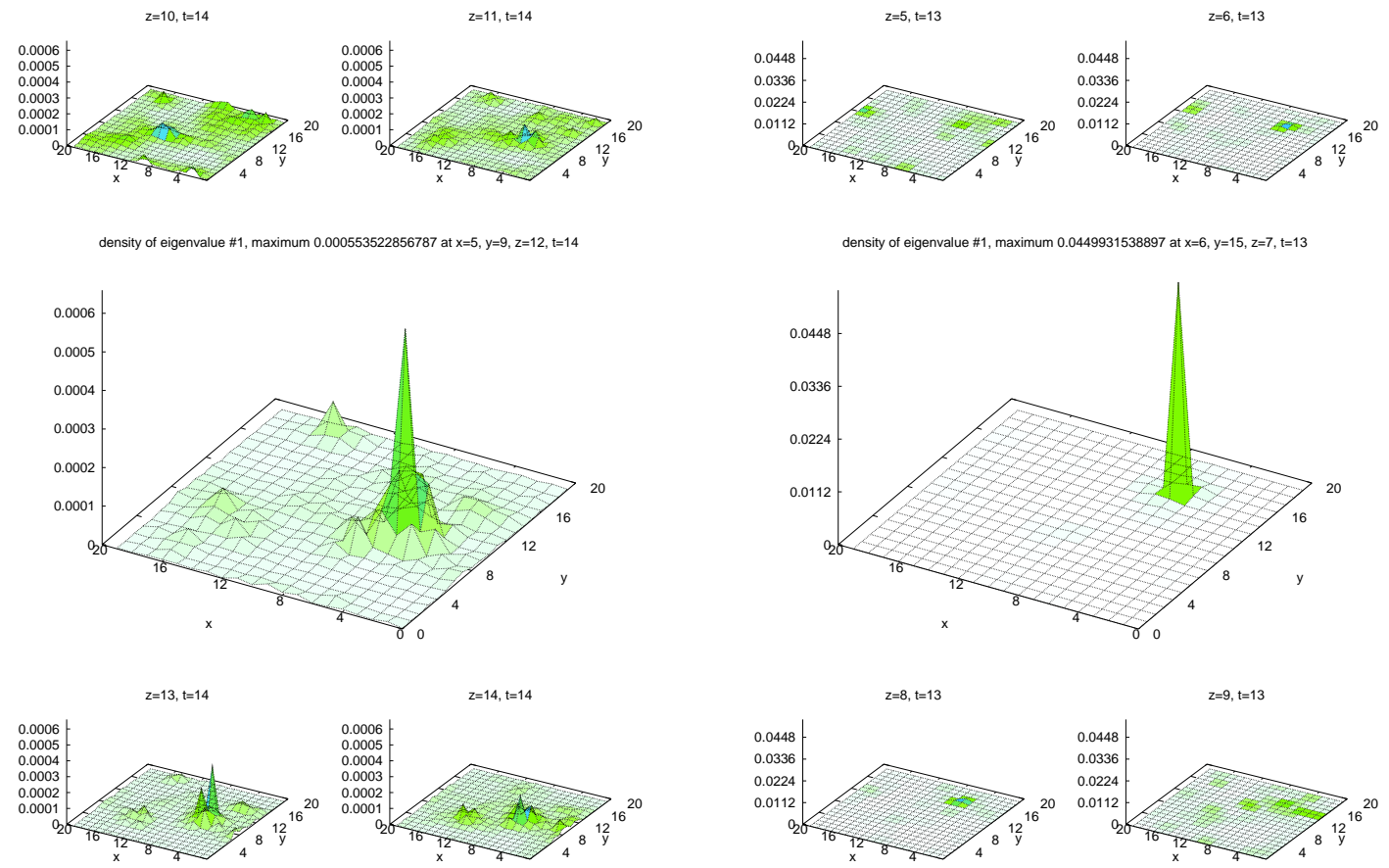

Figure 5: Maximum density peak (center) of the first asqtad eigenmode on a $20^{4}$-lattice at $\beta_{L W}=3.3$ with upper (above) and lower (below) z-slices of the same t-slice. Eigenmodes are computed on (full (left) and center-projected (right) lattices (notice different scales!).
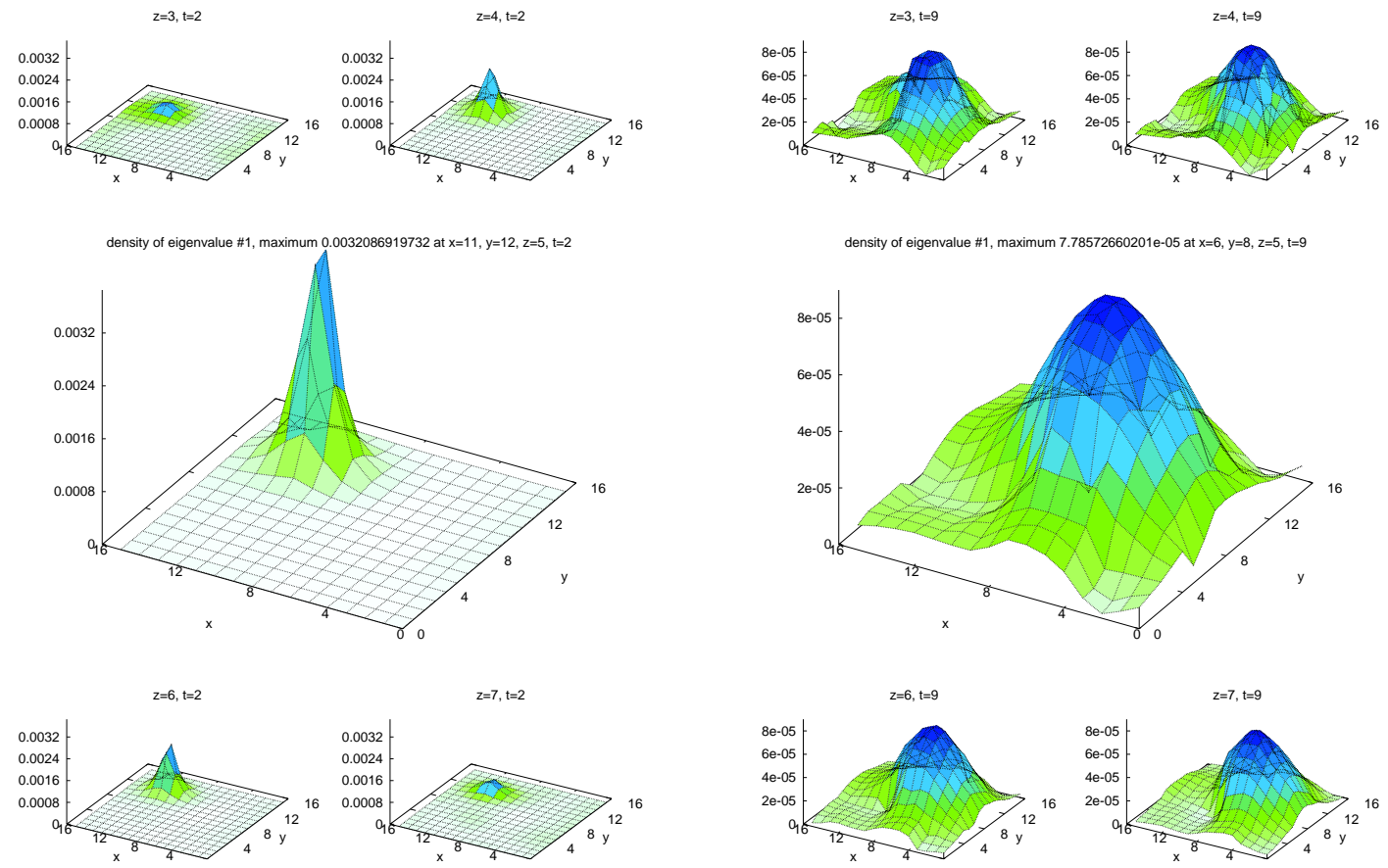

Figure 6: Maximum density peak (center) of the first overlap eigenmode on a $16^{4}$-lattice at $\beta_{L W}=3.3$ with upper (above) and lower (below) z-slices of the same t-slice. Eigenmodes are computed on (a) full lattices, and (b) center-projected lattices (notice different scales!). 


\section{Conclusions}

We find that the thin vortices found in center projection give rise to a low-lying spectrum of near-zero Dirac eigenmodes, provided that the chiral symmetry of the Dirac operator does not depend on the smoothness of the lattice configuration. Thus, the vortex excitations of the vortex-only lattice carry not only the information about confinement, but are also responsible for $\chi S B$ via the Banks-Casher relation. There are significant correlations between center vortices and the low-lying modes of both the asqtad and overlap Dirac operators. These eigenmodes have their largest concentrations in point-like regions, rather than on submanifolds of higher dimensionality. Taken together, correlations and dimensionality support the picture of a center vortex origin for topological charge, and indicate that center vortices have a strong effect on the properties of low-lying eigenmodes of the Dirac operator. A more detailed presentation is found in Ref. [14].

\section{References}

[1] A. Casher, Phys. Lett. B83 (1979) 395.

[2] J. Gattnar, C. Gattringer, K. Langfeld, H. Reinhardt, A. Schäfer, S. Solbrig and T. Tok, Nucl. Phys. B 716 (2005) 105 [arXiv:hep-lat/0412032].

[3] C. Gattringer, Phys. Rev. D 63 (2001) 114501 [arXiv:hep-lat/0003005];

C. Gattringer, I. Hip and C. B. Lang, Nucl. Phys. B 597 (2001) 451 [arXiv:hep-lat/0007042].

[4] T. Banks and A. Casher, Nucl. Phys. B169 (1980) 103.

[5] R. Narayanan and H. Neuberger, Nucl. Phys. B443 (1995) 305 [arXiv:hep-th/9411108]; H. Neuberger, Phys. Lett. B417 (1998) 141 [arXiv:hep-lat/9707022].

[6] Kostas Orginos, Doug Toussaint and R.L. Sugar, Phys. Rev. D 60 (1999) 054503 [arXiv:hep-lat/9903032]; G.P. Lepage, Phys. Rev. D 59 (1999) 074502 [arXiv:hep-lat/9809157].

[7] C. Alexandrou, P. de Forcrand and M. D’Elia, Nucl. Phys. A 663 (2000) 1031 [arXiv:hep-lat/9909005].

[8] F. V. Gubarev, S. M. Morozov, M. I. Polikarpov and V. I. Zakharov, [arXiv:hep-lat/0505016].

[9] V. G. Bornyakov, E. M. Ilgenfritz, B. V. Martemyanov, S. M. Morozov, M. Müller-Preussker and A. I. Veselov, Phys. Rev. D 77 (2008) 074507 [arXiv:0708.3335 [hep-lat]].

[10] A. V. Kovalenko, S. M. Morozov, M. I. Polikarpov and V. I. Zakharov, Phys. Lett. B 648 (2007) 383 [arXiv:hep-lat/0512036].

[11] M. Engelhardt, Nucl. Phys. B 585 (2000) 614 [arXiv:hep-lat/0004013];

M. Engelhardt and H. Reinhardt, Nucl. Phys. B 567 (2000) 249 [arXiv:hep-th/9907139].

[12] M. Lüscher and P. Weisz, Phys. Lett. B 158 (1985) 250.

[13] H. Leutwyler and A. Smilga, Phys. Rev. D 46 (1992) 5607.

[14] R. Höllwieser, M. Faber, J. Greensite, U.M. Heller and Š. Olejník, Phys. Rev. D 78 (2008) 054508 [arXiv:0805.1846 [hep-lat]]. 